\title{
Agôn
}

Revue des arts de la scène

1 | 2008

Interstices, entractes et transitions

\section{L'opéra du dialogue (impossible ?) des cultures}

Zaïde - Adama de W. A. Mozart, C. Czernowin et C. Guth ou l'art de l'agencement, de l'interstice et de la transition

David Marron

\section{(2) OpenEdition}

Journals

Édition électronique

URL : http://journals.openedition.org/agon/723

DOI : 10.4000/agon.723

ISSN : 1961-8581

Éditeur

Association Agôn

Référence électronique

David Marron, «L'opéra du dialogue (impossible ?) des cultures », Agôn [En ligne], 1 | 2008, mis en ligne le 28 mai 2020, consulté le 02 juin 2020. URL : http://journals.openedition.org/agon/723 ; DOI : https://doi.org/10.4000/agon.723 


\title{
L'opéra du dialogue (impossible ?) des cultures
}

\author{
Zaïde - Adama de W. A. Mozart, C. Czernowin et C. Guth ou l'art de \\ l'agencement, de l'interstice et de la transition
}

\author{
Zaïde, Gomatz : \\ Möchten doch einst Ruh' und Friede \\ Nach so vieler Qual und Pein \\ unsrer Treue Preise sein !"
}

Johann Andreas Schachtner, Zaïde

Durant l'été 2006, le Festival de Salzbourg célébrait les 250 ans de la naissance de Wolfgang Amadeus Mozart. Pour cette édition particulière, tous les opéras du compositeur furent donnés et confiés, majoritairement, à des metteurs en scène allemands et autrichiens. ${ }^{2}$ Le parti pris de Peter Ruzicka, alors directeur du Festival, ne fut pas de rendre un hommage convenu à Mozart à travers des mises en scène classiques mais, bien au contraire, de donner la possibilité à de nouvelles visions scéniques et musicales de voir le jour, de (re)mettre en jeu ces œuvres afin d'en révéler de nouvelles aspérités. C'est dans ce cadre que la compositrice israélienne Chaya Czernowin fut conviée à composer, pour la salle à l'italienne du Landestheater de Salzbourg, une œuvre lyrique en relation avec Zaïde (K. 344), un singspiel ${ }^{3}$ inachevé de Mozart. ${ }^{4}$ Plus précisément, le projet Zaïde - Adama, mis en scène par Claus Guth, donne à voir et à entendre deux opéras enlacés, mettant en œuvre une poétique de la relation scénique, au carrefour de l'humain, de l'art et de la société, une réflexion sur le désir de dialogue avec l'autre mais aussi sur la puissance des différences culturelles, religieuses et du poids communautaire. ${ }^{5}$

On s'intéressera donc au statut particulier de l'opéra de Mozart et aux ouvertures qu'il offre à chaque nouvelle production mais aussi au tissage des deux oeuvres Zaïde - Adama, aux zones de frottements et d'interpénétrations textuelles, musicales et scéniques. Ce sera

\footnotetext{
${ }^{1}$ Zaïde, Gomatz : « Puissent un jour la sérénité et la paix / récompenser notre fidélité / après tant de tourments et de peines !» (Trad. : Isabelle Demmery)

${ }^{2}$ Hormis Zaïde - Adama, Claus Guth a mis en scène, pour cette édition 2006 du Festival de Salzbourg, Les Noces de Figaro, sous la direction musicale de Nikolaus Harnoncourt.

${ }^{3}$ « Par opposition à l'opéra en italien, l'opéra allemand tel que Mozart le pratique mêle au chant des dialogues parlés, et non des récitatifs. [...] Sous l'influence de l'opéra italien, le singspiel intègre des airs virtuoses, des ensembles complexes, des chœurs : Mozart porte le genre à un niveau de raffinement musical et de richesse expressive qu'il n'avais pas connu avant lui », in Pierre Michot, Mozart, opéras mode d'emploi. Paris : L'AvantScène Opéra, Editions Premières Loges, 2006, p. 51.

${ }^{4}$ Consciente de l'honneur d'une telle proposition mais aussi du risque que cela représentait, la compositrice prit néanmoins deux semaines de réflexion avant de donner une réponse positive à l'invitation de Peter Ruzicka. Une fois le projet mis en route, il fallut deux ans de travail et de maturation pour arriver à la version finale de 2006.

${ }^{5}$ Wolfgang Amadeus Mozart / Chaya Czernowin, Zaïde - Adama. Deutsche Grammophon, M 22, DVD 073 4252, 2006.
} 
l'occasion de réfléchir sur ce qui se joue dans les interstices de cette totalité hybride, que ce soit lors du passage de tel numéro de l'opéra de Mozart à celui de Czernowin, que ce soit lors de certaines actions scéniques ou lors du moment central de l'entracte et ses incidences sur le dispositif scénique et les rapports de force entre personnages.

\section{Zaïde ou l'aventure d'une création musicale posthume}

Si L'Enlèvement au sérail, singspiel se déroulant dans un cadre oriental, a longtemps été une œuvre de Mozart particulièrement prisée des amateurs d'opéra, Zaïde, ou le Sérail, ayant pourtant un cadre similaire, n'a pas toujours eu la même fortune. Cependant, des liens sensibles apparaissent entre les deux oeuvres. Comme le mentionne Pierre Michot, outre le décor de turquerie et l'évasion ratée, on trouve «la figure du musulman que l'on croit intraitable et qui finit par donner aux chrétiens une leçon de tolérance et d'humanité »». ${ }^{6}$ Cependant, la tonalité des livrets est quelque peu différente d'une œuvre à l'autre. Lorsque Mozart a le projet de terminer Zaïde pour le faire jouer à Vienne, il lui est signalé que le traitement du sujet est peut-être trop sérieux pour le goût du public. ${ }^{7}$ Loin d'être une œuvre légère ou exotique, Zaïde se présente comme une comédie sentimentale dans laquelle le sérieux est bel et bien de rigueur. C'est donc au profit de L'Enlèvement au Sérail que Mozart abandonne sa première histoire de Sérail.

Composé entre 1779 et 1780, sur un livret de Johannes Andreas Schachtner, d'après Joseph Sebastiani, Zaïde ne vit le jour sur scène que le 27 janvier 1866, à l'Opéra de Francfort, soit une cinquantaine d'années après la mort de Mozart. D'ailleurs, c'est en rangeant les papiers de son mari que Constance découvre le manuscrit d'un opéra en deux actes, inachevé et sans titre. Cependant, l'œuvre ne sera publiée qu'en 1838 et baptisée alors Zaide, oder Das Serail, par l'éditeur J. A. André. Le livret de Schachtner s'inspire d'un singspiel, mis en musique par Joseph von Frieberg, intitulé Le Sérail, ou la Rencontre imprévue d'un père, d'une fille et d'un fils réduits en esclavage, dans lequel un chrétien, Gomatz, et une jeune Européenne, Zaïde, sont prisonniers du sultan Soliman. Tous deux s'évadent, aidés par Allazim. Finalement, Soliman pardonne cette évasion et leur accorde la liberté. Dans l'opéra de Mozart, on ne sait pas exactement ce qu'il se passe à la fin puisque le compositeur n'a pas mis un terme à sa partition, laissant en suspens l'ultime dénouement. Zaïde et Gomatz vont-ils mourir ? Vont-ils être finalement épargnés ? Nul ne le sait.

Cette destinée particulière de l'œuvre inachevée peut expliquer en partie la relative distance du public, des institutions mais aussi celle des interprètes. Néanmoins, l'œuvre revient sur le devant de la scène progressivement et fait l'objet de plusieurs enregistrements et mises en scène durant la seconde moitié du $\mathrm{XX}^{\mathrm{e}}$ et en ce début de $\mathrm{XXI}^{\mathrm{e}}$ siècle. Aussi la question de l'unité, de l'agencement et de la réalisation finale de l'œuvre se présente à un moment ou à un autre aux interprètes et aux metteurs en scène. Comment l'opéra est-il traité lorsqu'il est porté à la scène ou qu'il fait l'objet d'un nouvel enregistrement ? Que faire donc avec une telle partition inachevée?

\footnotetext{
${ }^{6}$ Pierre Michot, Mozart, opéras mode d'emploi, op. cit., p. 96.

${ }^{7}$ Id.
} 


\section{Quel devenir pour une partition inachevée?}

D'autres œuvres de Mozart peuvent servir à compléter Zaïde. C'est le cas notamment dans la production dirigée par Louis Langrée et mise en scène par Peter Sellars, à Vienne en 2006 et à Aix-en-Provence en 2008, où les passages parlés de l'œuvre sont supprimés, remplacés par des textes parlant de l'esclavage dans la société contemporaine ou par des musiques orientales. Cette version emprunte l'ouverture ainsi que les interludes à la musique de scène de Mozart Thamos, roi d'Egypte contemporaine de celle de Zaïde. Dans la production dirigée par Nikolaus Harnoncourt, c'est la Symphonie $n^{\circ} 26$ de Mozart qui sert d'ouverture. Alternativement avec la musique de Mozart, Tobias Moretti, un acteur autrichien à succès ${ }^{8}$, y déroule aussi « un flot de paroles », dont il est l'auteur, sur l'action de l'opéra et commente aussi le contexte de la création mozartienne, le tout mis en parallèle avec les débats actuels sur la tension entre l'Orient et l'Occident, la tolérance et la violence, et agrémenté de références à Nietzsche, à Elfriede Jelinek, au Faust de Goethe ou encore au Don Carlos de Schiller. Moretti allonge ainsi de façon notable l'œuvre et, d'une certaine manière, accentue le désir qu'avait Mozart d'intégrer plus de paroles dans le genre lyrique. D'ailleurs, la parole est déjà présente, de façon significative, à travers les deux mélodrames, ou les deux mélologues comme indiqué dans la partition, les numéros 2 et $9 .{ }^{9}$

Dans d'autres occasions, commande est passée à un compositeur contemporain. C'est le cas lorsqu'en 2006, Bernard Cavanna compose un opéra en cinq mouvements d'une cinquantaine de minutes intitulé Zaïde Actualités, destiné à être donné conjointement avec l'œuvre de Mozart lors d'une même soirée intitulée Zaïde(s), mise en scène par Michel Rostain, également initiateur du projet. $\mathrm{Si}$, dans cette production, Zaïde Actualités précède Zaïde, l'opéra de Cavanna réapparaît cependant dans une reprise finale. Le metteur en scène a remanié le livret en supprimant notamment les récitatifs au profit de dialogues en français, toujours ponctués par l'orchestre, et en réalisant un croisement du français et de l'allemand dans les parties chantées. Ainsi, à travers le regard d'un journaliste, Zaïde Actualités raconte la version actuelle du drame de la jalousie en jeu dans l'opéra de Mozart. ${ }^{10}$ Zaïde y est tuée par son mari Slimane. C'est aussi l'occasion d'une mise en perspective de la fascination du monde contemporain pour les faits-divers, ces drames présents quotidiennement dans les journaux, à la télévision, à la radio ou sur internet.

Adama s'inscrit ainsi dans le cas de figure d'une création contemporaine, dialoguant avec Zaïde. Cependant, la relation entre l'œuvre de Mozart et celle de Czernowin est plus complexe que dans le cas de Zaïde(s), du fait même du tissage continu, et non ponctuel, des

\footnotetext{
${ }^{8}$ Il est notamment connu pour son interprétation du commissaire Richard Moser, durant les années 90, dans la série télévisée germano-autrichienne Kommissar Rex.

${ }^{9}$ Voici ce que disait, le 12 novembre 1778, Mozart, alors sous l'influence des œuvres de Benda, à propos de son goût pour la parole à l'opéra : «Vous savez qu'on n'y chante pas, mais qu'on y déclame, et que la musique est comme un récitatif obligé. Par moments, on parle aussi sur la musique, et cela fait la plus magnifique impression. [...] Savez-vous qu'elle est ma manière de voir ? On devrait traiter de cette façon la plupart des récitatifs dans l'opéra, et ne chanter que de temps en temps ces récitatifs, lorsque les paroles sont bonnes à rendre en musique », cité par Pierre Michot, Mozart, opéras mode d'emploi, op. cit., p. 97.

${ }^{10}$ Extrait du dossier de presse du spectacle Zaïde(s) : «Un journaliste commence par donner l'information : “Le cadavre de Zaïde a été retrouvé ce matin”. Ici, pas de miracle, la trop banale violence conjugale. Une série de flash-back rappelle le cours inéluctable des événements : l'amour de Zaïde et de son mari, Slimane, au début de leur union; les liens du couple qui peu à peu se sont distendus ; les mésententes, les menaces puis les coups. Zaïde qui finit par s'enfuir. La violence de Slimane qui ne peut imaginer perdre Zaïde, qui la roue de coups et la tue dans un terrain vague, derrière un supermarché. Le journaliste interviewe Slimane pour raconter au public cette horreur. Slimane chante l'amour exclusif qu'il avait pour la femme qu'il vient de tuer ».
} 
œuvres.

\section{Chaya Czernowin et Claus Guth : avant Zaïde-Adama, le projet Pnima... ins Innere}

Zaïde - Adama n'est pas cependant la première incursion de Chaya Czernowin dans le domaine de l'opéra contemporain. En 2000, fut donné à la biennale de Munich, à l'invitation de Ruzicka, Pnima... ins Innere ${ }^{11}$, son premier opéra, basé sur le roman Voir ci-dessous : Amour de l'auteur israélien David Grossman. ${ }^{12}$ Un vieil homme revenu des camps de concentration et son petit-fils y tentent de se rencontrer, de tisser des liens dans un espace étrange, hors-temps, qui n'est autre qu'une grande pièce vide aux murs délavés, dans la mise en scène de Claus Guth et la scénographie de Christian Schmidt, que nous retrouverons pour la création de Zaïde - Adama. ${ }^{13}$ Des images filmées, plus ou moins abstraites, y sont aussi projetées par moments. De plus, il n'y a pas à proprement parler de livret dans cette œuvre, ce qui lui confère une dimension mystérieuse supplémentaire. ${ }^{14}$ Tout semble se jouer dans un temps en suspension, un " au-delà des mots », où les corps tentent de se rencontrer, dans une « esthétique du peu ». Ainsi, avant Zaïde - Adama, Pnima... ins Innere travaillait déjà les possibles et les marges du genre lyrique.

D'ailleurs, certaines résonances musicales et scéniques sont présentes entre Pnima... ins Innere et Adama, notamment dans le traitement musical et dramaturgique du désir de relation et la question de la présence contraignante ou traumatisante. Dans Pnima... ins Innere, il s'agit de la Bête cachée dans la cave, du souvenir présent de l'horreur nazie. Dans Adama, un Palestinien et une Israélienne s'approchent, se disent des mots d'amour, se rejettent et sont rattrapés par la force des deux communautés opposées, une frontière les séparant finalement. Leur histoire finira dans la violence et la tristesse. Ainsi, dans les deux opéras de Czernowin, la question de la relation est au cœur des œuvres. Mais justement comment une œuvre comme Adama, d'une terrible rugosité musicale et dramatique, jouant sur le « presque rien » musical et vocal, arrive-t-elle à s'articuler avec le lyrisme mozartien de Zaïde?

\section{Zaïde - Adama, un opéra hybride sur la rencontre et la relation}

Zaïde - Adama est donc composé de deux opéras indépendants, avec l'orchestre mozartien dans la fosse et l'orchestre contemporain en fond de scène. Lors de la première salzbourgeoise, l'orchestre du Mozarteum sous la direction d'Ivor Bolton jouait, dans la fosse,

\footnotetext{
${ }^{11}$ Pnima... ins Innere a reçu le prix récompensant la meilleure création lyrique contemporaine de l'année 2000 attribué par la revue Opernwelt, a remporté, la même année, le Bayerischer Theater Prize et a fait l'objet d'une édition en DVD chez Mode Records (Mode 169), en 2006.

${ }^{12}$ David Grossman, Voir ci-dessous : Amour (traduit de l'hébreu par Judith Misrahi et Ami Barak). Paris : Le Seuil, coll. « Points », 1991.

${ }^{13}$ Dans cet espace, le vieil homme et l'enfant sont interprétés par des rôles muets alors que les chanteurs sont invisibles des spectateurs, interprétant leurs parties vocales hors-scène. La présence physique des deux personnages fut pensée par le metteur en scène, convaincant la compositrice, d'abord réticente à toute incarnation scénique, de ce choix.

${ }^{14}$ Plus précisément, Pnima ... ins Innere est basé sur Momik, la première partie du roman, centrée sur la relation entre le vieil homme et l'enfant.
} 
la musique de Mozart, tandis que l'Österreichisches Ensemble für Neue Musik sous la direction de Johannes Kalitzke, soutenu par l'Experimentalstudio für akustische Kunst de Fribourg, interprétait sur scène la composition de Czernowin. Les choristes des Basler Madrigalisten faisaient aussi partie de l'aventure.

D'une durée totale de cinquante-cinq minutes, Adama, « terre » en hébreu, se distingue des précédentes tentatives entreprises pour compléter les soixante-quinze minutes de Zaïde, car elle revendique une entière autonomie, même si le texte d'Adama, rédigé par la compositrice elle-même, est basé sur quelques mots-clés tirés du livret de Schachtner. Ainsi, Adama peut se définir comme « une histoire d'amour entre une Israélienne et un Palestinien, où la peur et le poids moral des communautés dominent vite, incarnés par la figure archétypale du Père, figure toute puissante, qui symbolise la tradition et l'immobilisme $»{ }^{15}$ S'il y a une autonomie des œuvres, cela n'exclut pas les proches relations.

Le metteur en scène Claus Guth et le scénographe Christian Schmidt, en charge aussi des costumes, ont conçu un dispositif scénique unique pour les deux œuvres. Il s'agit d'un espace où l'on trouve un bureau, une chaise en formica ainsi qu'un radiateur imposant. Une double porte vitrée, aux dimensions importantes, se trouve aussi en fond de scène. Une des vitres est brisée et laisse entrevoir l'orchestre contemporain, disposé derrière le décor, dans une pièce adjacente. Cette double porte vitrée, ouverte ou fermée selon le moment de l'œuvre, est un élément important dans cette scénographie du dialogue et/ou conflit des cultures. La lumière est plutôt blafarde dans cet espace aux murs blanchâtres. Ce lieu ainsi créé apparaît comme issu du « quotidien », avec son mobilier familier. Les chanteurs des deux productions y évoluent, concourant ainsi à créer le lien à vue entre les deux œuvres, un continuum visuel mettant en relation directe les interprètes des deux productions. On est donc loin d'une quelconque mise en scène orientalisante, évoquant le Sérail du sultan Soliman. Les spectateurs peuvent ainsi appréhender l'œuvre avec une certaine distance et porter un regard nouveau sur la portée profonde de l'opéra de Mozart.

La compositrice n'a pas voulu compléter le singspiel, en composant à la manière de Mozart, ni même en reprenant les personnages de Zaïde. Elle a préféré l'intégration de ses propres éléments musicaux entre les numéros de Mozart, en travaillant d'autres situations et d'autres personnages. Ainsi, les fragments ou numéros de chacun des deux opéras alternent de façon presque régulière. D'ailleurs, c'est sur la musique de Czernowin que s'ouvre l'œuvre, qui se referme sur celle de Mozart. La compositrice résoud ainsi la question de l'ouverture, manquante dans Zaïde, sans toutefois clore l'œuvre, gardant intact le mystère de l'inachevé laissé par Mozart. Le sens reste aussi de la sorte ouvert.

Durant l'ouverture, des vidéos de passants, de soldats et du mur séparant Israël et la Palestine sont projetées, le tout montré de manière allusive, sans insister sur tel ou tel territoire. Cependant le cadre est bel et bien posé. Dès les premiers instants de l'œuvre, il apparaît qu'il sera question d'un drame profondément actuel, d'individus pris au cœur d'enjeux qui les dépassent, au cœur d'une tension insoutenable et meurtrière. L'Homme et la Femme d'Adama, respectivement palestinien et israélienne, représentent chacun le cri profond de leur peuple, au-delà de la simple anecdote de leur histoire personnelle. Leur histoire, c'est celle d'une région plongée dans une crise fondamentale et, pour l'instant, sans issue. Cependant, la compositrice a essayé d'éviter l'écueil d'une œuvre trop manichéenne.

\footnotetext{
${ }^{15}$ Julia Spinola, « En plein conflit des cultures » (trad. d'Odile Demange), in Zaïde-Adama, op. cit., p. 15.
} 
Pour mettre en évidence ce qui se joue dans le passage d'un régime musical à l'autre, on s'arrêtera sur deux extraits pris dans le premier et le deuxième acte. Ces deux extraits donnent à voir notamment l'entrée en scène d'Allazim, personnage des plus importants dans l'opéra de Mozart puisqu'il est l'homme qui encourage Zaïde et Gomatz à fuir mais qui est aussi la voix prônant la réconciliation entre les parties adverses. En quelque sorte, il représente à lui seul l'esprit traversant le projet, le personnage médiateur, le trait d'union, à l'image de celui présent dans le titre Zaïde - Adama.

\section{Le souffle de la liberté et le grondement de la catastrophe}

Le premier extrait choisi se trouve dans la dernière partie de l'Acte I. Nous nous arrêterons sur la séquence donnant à voir et à entendre l'enchaînement des numéros 6 et 7 de Zaïde, avec, intercalé entre les deux, un passage d'Adama. Tout d'abord, dans le sixième numéro de l'opéra de Mozart, Gomatz, ivre d'amour, remercie son Dieu de lui avoir permis de rencontrer Zaïde. Ensuite, la Femme et l'Homme, le couple d'Adama, expriment leur contentement, avec des expressions verbales très proches, à travers les termes signifiant « soleil » et « ciel », en hébreu et en arabe. Cette partie centrale s'intitule justement Sonne. ${ }^{16}$ Lorsque vient le septième numéro de Zaïde, Allazim, un proche du sultan, encourage le couple et promet aux amoureux de les aider à fuir.

Durant l'air de Gomatz, les deux couples sont sur le plateau. Un personnage portant un masque jaune imposant occupe aussi l'espace scénique. La présence d'un personnage masqué n'est pas nouvelle dans le travail scénique de Claus Guth. On la retrouve notamment dans sa mise en scène d'Iphigénie en Tauride de Christoph Willibald Gluck, pour l'Opernhaus de Zurich, en 2001. Les personnages y étaient «prisonniers d'une sphère marquée par des liens familiaux déchirés, des angoisses, des traumatismes et un prédéterminisme, qui finit par aboutir à leur délivrance ${ }^{17}{ }^{17}$ Le metteur en scène déclare à ce propos : " J'ai plusieurs fois utilisé ce système de masques afin de donner une forme tangible à l'état psychologique des personnages $\gg .^{18}$ En tombant le masque, les personnages se libèrent ainsi progressivement des entraves et retrouvent le chemin de la vie. Dans Zaïde - Adama en revanche, la puissance du masque reste entière jusque dans les tout derniers instants, comme si la figure du Pouvoir ne pouvait céder le pas à l'humain, ses doutes et sa magnanimité.

Ainsi, cette figure étrange, assise sur un radiateur monumental, en fond de scène, depuis le début de l'œuvre, semble surveiller les deux couples, maîtriser du regard leurs agissements. Sa descente du radiateur et son arrivée sur le plateau coïncident donc avec la joie de Gomatz. Comme pour briser cet espoir de liberté naissant, ce personnage aligne les pierres qui se trouvent en vrac sur le plateau, formant une frontière, coupant l'espace en deux, séparant l'Homme et la Femme. Cette ligne n'est pas sans évoquer le mur de séparation entre Israël et Palestine, le mur de la peur, la barrière de la relation impossible. Zaïde et Gomatz sont eux aussi séparés par cette frontière. La musique de Czernowin est d'une intense aridité. Elle se déroule par bribes, presque figée. Les mots et les sons semblent se dérober et se dilater dans un hors-temps musical et dramatique. C'est à ce moment-là qu'Allazim fait son entrée en scène, avec le retour de la musique rayonnante de Mozart.

\footnotetext{
${ }^{16}$ Sonne : Soleil.

${ }^{17}$ Wolf Christian Fink, « Qu'est-ce qu'un opéra sans drame ? », in Livret d'Iphigénie en Tauride. Arthaus Musik, DVD 100 376, 2001.

${ }^{18}$ Claus Guth, cité dans le livret d'Iphigénie en Tauride. Arthaus Musik, DVD 100 376, 2001.
} 
Cette arrivée est des plus importantes. Le texte qu'il chante alors n'est autre qu'un hymne à la liberté et à la révolte :

\author{
Allazim : \\ Nur mutig, mein Herze, versuche dein Glück! \\ Verschaffe dir selber ein bessers Geschick! \\ Man muß nicht verzagen, \\ durch tapferes Wagen \\ schlägt oftmals der Schwache den Stärkern zurück. ${ }^{19}$
}

Chaya Czernowin accentue la force du propos d'Allazim en faisant se superposer, durant quelques instants, la musique de Zaïde et sa propre musique. Un parasitage est nettement perceptible, donnant à entendre une musique du troisième type, absolument inouïe, mêlant consonances et dissonances. Comme le note Julia Spinola, « la compositrice aime ces enchevêtrements, ce travail avec des strates hétérogènes qui se heurtent et se frottent pour produire la forme musicale $\gg{ }^{20}$ Cette superposition des musiques va trouver un écho dans l'action scénique d'Allazim. Ce dernier, situé sur l'alignement des pierres, à mi-chemin entre les personnages des deux opéras, enlève un des blocs de pierre, créant ainsi une brèche dans le « mur ». Allazim apparaît alors comme le personnage de l'entre-deux, de l'interstice possible dans lequel va s'engouffrer le vent de la liberté. Il ouvre ainsi la possibilité d'un horizon lumineux, celui d'une rencontre possible. Ce n'est pas étonnant alors s'il chante : " Sehet dort in sanften Wogen, wie der bunte Regenbogen / euch als Friedensbote lacht $»^{21}$, des paroles annonciatrices d'un avenir pacifique.

Cependant, le premier acte ne se termine pas sur la même tonalité pour les deux œuvres. Si la toute fin de l'acte n'est autre que le huitième numéro de Zaïde où les amoureux décident de fuir ensemble, aidés par Allazim, sous le regard impuissant du personnage masqué, le passage d'Adama, précédant ce moment-là, intitulé Erde, Blut, Mutter ${ }^{22}$, se clôt sur la résurgence d'une haine ancestrale, après un rapprochement des corps de l'Homme et de la Femme. Les mots « terre », «sang » et « mère » semblent alors constituer métaphoriquement une barrière entre le couple d'Adama, les éloignant progressivement. La partie instrumentale est absente quelques instants, mettant particulièrement en avant les voix. Celles-ci deviennent de plus en plus tendues au fur et à mesure de leur éloignement Chacun des deux personnages s'assoit sur une chaise, d'un côté et de l'autre de la frontière coupant le plateau en deux. Euxmêmes ne se regardent plus et semblent fondamentalement étrangers l'un à l'autre. De plus, des images du mur séparant Israël et la Palestine sont alors projetées sur le mur de fond de scène. La scène se donne alors à voir comme le reflet d'une relation impossible, sans issue.

Cet acte se finit donc sur une lueur d'espoir - l'arc-en-ciel - mais aussi sur l'annonce de la catastrophe - l'orage qui gronde, la couleur sang -, comme nous pouvons l'entendre dans les propos d'Allazim et de Zaïde dans le trio final :

\title{
Allazim : \\ Sehet dort in sanften Wogen, \\ wie der bunte Regenbogen \\ euch als Friedensbote lacht.
}

\footnotetext{
${ }^{19}$ Allazim : « Courage mon cœur, tente ta chance ! / Euvre toi-même à un meilleur destin ! / Il ne faut pas désespérer, / par une audace intrépide, / le faible peut souvent repousser le fort. » (trad. : Isabelle Demmery).

${ }^{20}$ Julia Spinola, « En plein conflit des cultures » (trad. : Odile Demange), in Zaïde-Adama, op. cit., p. 15.

${ }^{21}$ Allazim : « Voyez là-bas sur les vagues légères, / un arc-en-ciel vous sourit / comme un messager de paix ». (trad. : Isabelle Demmery)

${ }^{22}$ Erde, Blut, Mutter : Terre, sang, mère.
} 
Zaïde :

Aber seht dort in der Ferne

blutige Kometensterne!

Hört ihr, wie der Donner kracht ? ${ }^{23}$

\section{La vengeance communautaire comme horizon}

Entre les deux actes, le décor ne subit pas de changements notables si ce n'est celui de la dimension de la table et de la chaise en formica. Celles-ci deviennent imposantes, inhumaines d'une certaine façon, faisant apparaître les personnages dans un environnement qui les dépasse, dans une situation qui n'est pas à la mesure de l'homme. Ainsi, comme pour l'utilisation des masques dans le travail scénique de Claus Guth, la démesure concourt à révéler un état psychologique, une tension se jouant sur le plateau ou dans les esprits. ${ }^{24}$

Le deuxième acte s'inscrit donc sous le signe de la catastrophe annoncée à la fin du premier acte. Zaïde et Gomatz sont de retour dans le Sérail de Soliman, rattrapés après leur évasion. La figure patriarcale d'Adama donne voix, sur la musique de Mozart, aux propos de Soliman, lors du deuxième mélodrame de l'opéra, au tout début de cet acte. On notera qu'au moment de l'air de Soliman, c'est un des chanteurs de la production de Zaïde qui, à son tour, donne à entendre les propos du Sultan. D'ailleurs, lors du onzième numéro de Zä̈de, dans un air où il apparaît déterminé à rendre la justice dans le sang, Soliman bande les yeux de Zaïde et Gomatz mais aussi de l'Homme et de la Femme d'Adama. Une cause commune semble donc réunir Soliman et le Père : la vengeance suite à la trahison des deux couples.

La présence plus importante d'acteurs portant les grands masques jaunes révèle la place dominante de la force communautaire et de la vengeance durant le deuxième acte. D'ailleurs, lorsque Soliman, par exemple, enlève son masque, il se dévoile, montrant son vrai visage, ses émotions et ses faiblesses. Le doute peut alors surgir en lui. Lorsqu'il remet son masque, la communauté reprend ses droits. Ainsi, dans l'interstice du passage de la figure masquée à la figure démasquée semble se jouer la figure du pouvoir dans toute sa complexité, tiraillée entre ses principes et ses incertitudes, le dévoilement d'une ambivalence.

De façon évidente, cet acte se présente, dès les premiers instants, comme bien plus sombre et tendu, avec les figures traumatisantes de Soliman, du Père et de leur entourage. Pour annoncer la vengeance de Soliman et du Père envers Zaïde, Gomatz, l'Homme et la Femme, un mannequin habillé en femme est pendu sur scène. Ce mannequin est vêtu de la même robe que portent Zaïde et la Femme et sera maculé de sang. ${ }^{25}$ L'espace scénique devient alors aussi un lieu de contrastes de couleurs, le jaune vif côtoyant le rouge, le gris ou

\footnotetext{
${ }^{23}$ Allazim : « Voyez là-bas sur les vagues légères, / un arc-en-ciel vous sourit / comme un messager de paix ». Zaïde : « Mais voyez au loin / ces comètes couleur de sang ! Entendez-vous le tonnerre gronder ? ». (trad. : Isabelle Demmery)

${ }^{24}$ On retrouve notamment cette démesure des éléments dans la mise en scène du Fierrabras de Franz Schubert que Claus Guth réalisa pour l'Opernhaus de Zürich, en 2002. C'était alors un piano sur scène qui avait une taille imposante par rapport aux acteurs. D'ailleurs, dans Fierrabras, on retrouve aussi la thématique du personnage deus ex machina, la figure qui surveille le jeu ou qui le dirige, à travers l'incarnation en scène du compositeur Schubert. Le spectateur semble convié à entrer dans l'esprit de l'artiste lors de la composition de l'œuvre, à être au cœur de son cabinet de travail, avec les personnages sortant de son imaginaire.

${ }^{25}$ Du moins, ce sang de la vengeance, élément essentiel dans ce deuxième acte, est un colorant rouge utilisé par les acteurs. Ils se le versent aussi eux-mêmes à vu sur le corps. Ils font alors littéralement couler le sang, du moins le sang théâtral.
} 
encore le noir. La relative cohésion des couleurs de l'acte premier laisse place à une désunion marquée et " criante » de celles-ci, donnant à la scène une teinte chaotique et composite, à l'image des différences qui se jouent sur le plateau.

Dans le même ordre d'analyses, on remarquera que Gomatz et L'Homme sont habillés d'un pantalon noir et d'une chemise blanche alors que le Père et Soliman sont habillés d'un costume noir. L'aspect vestimentaire des hommes de pouvoir est donc normalisé, ceci étant accentué par la présence des masques identiques. Lorsque Soliman enlève sa cravate et la veste de son costume au début de l'acte II, furieux de la fuite de Zaïde, il se trouve habillé de la même manière que Gomatz et l'Homme israélien. Il y a alors une similitude troublante entre les deux camps opposés, ceux-ci apparaissant finalement identiques, comme frères. Claus Guth et Christian Schmidt ont ainsi conçu ce deuxième acte comme un jeu de contrastes mais aussi de correspondances, jouant sur les limites entre «norme» communautaire et « anormalité » des personnalités individuelles en devenir.

\section{Pardon et fraternité en question}

Après cette évocation de l'atmosphère chaotique du deuxième acte, nous allons nous arrêter plus particulièrement sur une séquence, dans la dernière partie de l'opéra, où les numéros 14 et 15 de Zä̈de, encadrent un passage d'Adama s'intitulant Tränen. ${ }^{26}$ Dans le quatorzième numéro, Allazim, également vêtu d'un costume, occupe une place de médiateur et implore la clémence du sultan Soliman. Vient ensuite Tränen, où les personnages de Zaïde et d'Adama se retrouvent, pour la première fois, ensemble sur scène. L'affliction est alors générale. Zaïde - Adama atteint un climax de la souffrance collective. Lorsque vient le quinzième numéro, Zaïde supplie le sultan d'épargner Gomatz et de la tuer, elle seule. La réponse de Soliman est alors sans équivoque puisqu'il ordonne la mort des deux amoureux.

Pour son entrée en scène, Allazim ouvre en grand les deux battants de la porte se trouvant en fond de scène. Par ce geste, il rompt déjà la séparation entre l'orchestre mozartien et l'orchestre contemporain, ce dernier apparaissant alors nettement. Allazim se présente comme celui qui ouvre les portes du dialogue et de la reconnaissance, au propre comme au figuré. D'ailleurs, les propos qu'il chante entrent également en résonance avec cette attitude de médiateur, de réconciliateur entre les parties ennemies alors que les deux couples sont sur le point de se faire égorger :

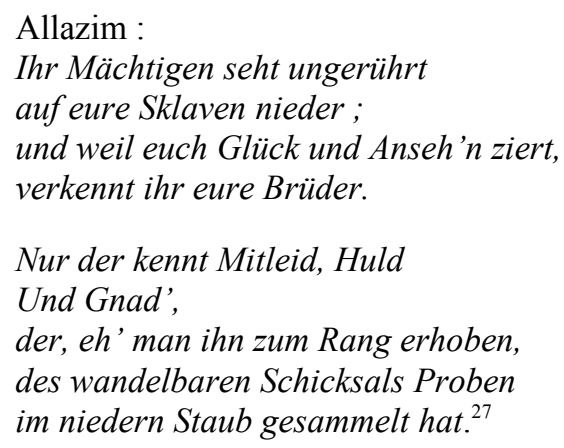

\footnotetext{
${ }^{26}$ Tränen : Larmes.

${ }^{27}$ Allazim : «O puissants, vous regardez / vos esclaves d'un œil indifférent ; / et parce que la chance et la renommée vous comblent, / vous méconnaissez vos frères. / Soliman : Seul connait la pitié, la clémence / et la grâce / celui qui, avec d'être élevé à un haut rang, / a connu les vicissitudes / qu'inflige la Fortune inconstante ». (trad. : Isabelle Demmery)
} 
Cependant, ce qui se joue sur scène n'est pas véritablement une reconnaissance mutuelle des parties. La musique des deux orchestres ne se superpose pas pour autant. Seule est présente celle de Zaïde, bien que l'orchestre contemporain soit visible. Cette alternance des musiques fait ressortir finalement le clivage apparent entre les deux communautés. Chaque clan semble camper sur ses positions, le mélange ne se fait pas. On notera qu'à ce moment précis, tous les personnages de Zaïde - Adama sont sur scène, rassemblés dans l'attente du dénouement heureux ou malheureux. Mais, si musicalement le mélange ne se produit pas véritablement, tous sont dans un territoire commun, échangent des regards, se rencontrent par le toucher. La chanteuse interprétant Zaïde développe alors une ligne vocale sur la musique de Czernowin. A ce moment-là, elle ne chante plus dans le style mozartien mais dans celui de la compositrice israélienne. Zaïde franchit alors une nouvelle frontière, arpente un nouveau territoire sonore. Soliman s'approche de la Femme israélienne et lui touche la main. Ce contact est l'occasion d'un passage de la musique de Czernowin à celle de Mozart, révélant le contact de deux corps étrangers, un échange de flux. Une certaine confusion domine alors dans un chaos scénique manifeste.

Lors du jugement final, Soliman et le Père remettent leur masque et sont les seuls à se tenir debout. On peut voir là, dans cette ultime scène, une représentation du pouvoir dans toute sa puissance inhumaine et inflexible, alors qu'Allazim tente de sauver une dernière fois les couples :

\author{
Allazim : \\ Mitleid, Herr, erhöre mich, \\ Mitleid, Herr, besänft' ge dich. \\ Soliman : \\ Fort, umsonst bemühst du dich, \\ geh', dein Fleh'n beleidigt mich. ${ }^{28}$
}

\title{
Zaïde - Adama ou l'acte d'amour lyrique
}

Le projet opératique de Chaya Czernowin et Claus Guth joue donc avec le passage d'un régime musical à l'autre, sans que le parasitage d'une œuvre par l'autre devienne systématique. S'il y a superposition, c'est toujours en relation avec un propos dramaturgique précis. De plus, lorsqu'elle élabore ces objets sonores hybrides, la compositrice connaît sans doute très bien les réticences qu'un tel agencement rencontrera chez les amateurs de Mozart, et plus largement d'art lyrique, notamment dans un lieu comme Salzbourg, berceau de la culture mozartienne. D'où l'enjeu d'une telle confrontation : faire de ce projet opératique, une oeuvre à part entière, où les deux opéras sont greffés ensemble sans qu'il soit possible de les séparer, du moins sans qu'Adama puisse être donné indépendamment. D'ailleurs, une fois l'expérience de Zaïde - Adama passée, une impression durable reste dans l'esprit du spectateur. Le passage de la fluidité de la musique de Mozart à la rugosité de la musique de Czernowin (et vice-versa), s'il ne semble pas naturel de prime abord, du fait même de la différence des textures instrumentales et vocales, apparaît comme évident à l'usage. Par un jeu de contraste, l'alternance des fragments permet de les mettre en valeur et fait ressurgir les particularités propres à chacune. Au lieu de se diluer dans l'agencement opéré, l'œuvre hybride gagne en force et en impact sur le spectateur.

\footnotetext{
${ }^{28}$ Allazim : « Pitié, seigneur, écoute-moi, / pitié, seigneur, radoucis-toi. »

Soliman : « Hors d'ici, tu t'obstines en vain, disparais, ta prière m'offense ». (trad. : Isabelle Demmery)
} 
Sans dénaturer fondamentalement l'œuvre mozartienne, le travail de Czernowin se présente comme une exploration sonore, radicalement éloignée de celle de Mozart mais révélant surtout, par un jeu de contrastes, l'étrange beauté d'une partition hybride, d'une grande complexité, qui tente de renouer des liens, de faire se rencontrer les " frères ennemis » (islam et christianisme, Israéliens et Palestiniens, musique classique et musique contemporaine,...) dans un acte d'amour lyrique.

David Marron

Docteur en Lettres et Arts, Spécialité Arts du spectacle, Université Stendhal - Grenoble 3. davmarron@gmail.com

\section{Références de l'article :}

David Marron, «L'opéra du dialogue (impossible ?) des cultures», Agôn [En ligne], L'interstice en scène, Dossiers, $\mathrm{N}^{\circ} 1$ : Interstices, entractes et transitions, mis à jour le : 15/12/2008, URL : http://agon.ens-1sh.fr/index.php?id=723. 